\title{
A Promoção da Saúde e a Prevenção Integrada dos Fatores de Risco para Doenças Cardiovasculares
}

\author{
The Promotion of Health and Integrated Prevention \\ of Risk Factors for Cardiovascular Diseases
}

Amanda Gomes Ribeiro ${ }^{1}$

Rosângela Minardi Mitre Cotta ${ }^{1}$

Sônia Machado Rocha Ribeiro ${ }^{1}$

${ }^{1}$ Departamento de Nutrição e Saúde, Universidade Federal de Viçosa. Av. Peter Henry Rolfs s/n, Campus Universitário. 36570-000 Viçosa MG.

rmmitre@ufv.br

\begin{abstract}
Cardiovascular diseases are currently the most common causes of morbimortality worldwide. In the context of disease prevention, actions creating favorable health environments and promoting healthy choices prove fundamental. Intervention programs with a community basis have been introduced in different countries since the beginning of the 1970s, aiming to modify cardiovascular risk factors and decrease morbidity and mortality due to cardiovascular diseases. In such experiences, the importance of public policies and cardiovascular risk vigilance systems needs to be stressed, permitting a more accurate assessment of the magnitude of the problem and the development of more cost-effective actions. The scope of this bibliographical study was to describe community-geared experiences in many countries in the campaign against cardiovascular diseases through health promotion and primary prevention of risk factors, as well as to identify in Brazil the vigilance actions of the risk factors of nontransmissible chronic diseases, stressing the strategic challenges facing the Family Health Program in tackling cardiovascular diseases.
\end{abstract}

Key words Health promotion, Disease prevention, Cardiovascular diseases, Risk factors, Family Health Program
Resumo As doenças cardiovasculares são, atualmente, as causas mais comuns de morbimortalidade no mundo. Na perspectiva da prevenção de doenças e agravos, tornam-se fundamentais ações que criem ambientes favoráveis à saúde e favoreçam escolhas saudáveis. Nesse contexto, programas de intervenção de base comunitária têm sido introduzidos em diferentes países, desde o início da década de 70, com o objetivo de modificar fatores de risco cardiovascular e diminuir a morbidade e a mortalidade por doenças cardiovasculares. Destaca-se nessas experiências a importância das políticas públicas e dos sistemas de vigilância dos fatores de risco cardiovascular, para conhecer a magnitude do problema e desenvolver ações mais custo-efetivas. Este estudo bibliográfico tem duplo objetivo: descrever experiências de diferentes países no combate às doenças cardiovasculares voltadas à comunidade através da promoção da saúde e da prevenção primária dos fatores de risco; e identificar no Brasil as ações de vigilância dos fatores de risco para as doenças crônicas não transmissíveis, ressaltando-se os desafios estratégicos impostos ao Programa de Saúde da Família no enfrentamento das doenças cardiovasculares. Palavras-chave Promoção da saúde, Prevenção de doenças, Doenças cardiovasculares, Fatores de risco, Programa Saúde da Família 


\section{Introdução}

As doenças cardiovasculares (DCV) são, atualmente, as causas mais comuns de morbidade e a principal causa de mortalidade em todo mundo. Anualmente a cardiopatia isquêmica, acidentes vasculares cerebrais, hipertensão arterial e outras cardiopatias são responsáveis por $15,9 \mathrm{mi}$ lhões de óbitos ${ }^{1}$.

No Brasil, assim como em outros países da América Latina, observou-se, nas últimas décadas, uma importante mudança no perfil da mortalidade da população, caracterizado pelo aumento dos óbitos causados por doenças crônicas não transmissíveis (DCNT). Dentre as DCNT, destacam-se as DCV e a hipertensão arterial (HA) com uma prevalência estimada de $35 \%$ na população acima de 40 anos. A instalação da HA ocorre cada vez mais precocemente e estima-se que cerca de $4 \%$ das crianças e adolescentes também sejam portadoras. Isso representa, em números absolutos, um total de 17 milhões de portadores da doença no país².

No Brasil, nos últimos anos, as DCNT vêm representando $69 \%$ dos gastos hospitalares no Sistema Único de Saúde (SUS), sendo as DCV responsáveis por alta frequência de internações. No ano de 2007, ocorreram 1.155.489 internações por doenças cardiovasculares, com custo global de R\$1.466.421.385,12 e um total de 91.182 óbitos ${ }^{3}$.

As mudanças nos padrões de morbimortalidade na população impõem, de forma constante, novos desafios, tanto para os gestores do setor da Saúde quanto para outros setores governamentais, cujas ações tenham repercussão na ocorrência dessas doenças ${ }^{4}$.

No ano de 2000, o Plano de Reorganização da Atenção à Hipertensão Arterial e ao Diabetes Mellitus no Brasil foi implantado pelo Ministério da Saúde, juntamente com outras organizações de saúde, com o objetivo de estabelecer diretrizes e metas para a reorganização no SUS para a atenção aos portadores dessas enfermidades, a partir da atualização dos profissionais da rede básica, do diagnóstico precoce do diabetes e da hipertensão e do encaminhamento dos pacientes diagnosticados às unidades de saúde para tratamento e acompanhamento. Uma das ações desse plano é a disponibilização para estados e municípios de um sistema informatizado - HiperDia - que permite o cadastramento e o acompanhamento dos portadores de HA e de Diabetes Mellitus (DM), enquanto que a médio prazo, permite o desenho do perfil epidemiológico desta populaçãa ${ }^{5}$.
O Plano de Atenção à Hipertensão e ao Diabetes e a implantação do HiperDia são importantes iniciativas para: o conhecimento da magnitude dessas doenças, o planejamento da aquisição de medicamentos e a capacitação profissional na rede de saúde brasileira. No entanto, "não suprem as demandas iminentes, por serem fragmentadas e centradas na assistência médica".

Nas DCNT existe a associação de fatores complexos e multivariáveis por se tratarem de doenças presentes no cotidiano dos indivíduos, por provocarem alterações objetivas e subjetivas em seu dia a dia e por serem, consequentemente, responsáveis por mudanças de comportamento ${ }^{6,7}$.

Os principais fatores ambientais modificáveis das DCV são os hábitos alimentares inadequados, o sedentarismo e a obesidade, associados às mudanças no estilo de vida como a cessação do tabagismo e o controle estresse psicoemocional ${ }^{8,9}$.

Dentro da prevenção, o monitoramento da prevalência dos fatores de risco para DCV, especialmente os de natureza comportamental, permitem, por meio das evidências observadas, a implementação de ações preventivas com maior custo-efetividade ${ }^{4}$.

No entanto, o padrão comportamental e os hábitos de vida estão estreitamente relacionados com condições objetivas de oferta, demanda, consumo, modismo e ainda as representações sociais da cultura e das relações sociais estabelecidas na sociedade ${ }^{10}$. Nesse contexto é que, além da prevenção primária, a promoção da saúde tem se configurado como alternativa teórica e prática para o enfrentamento global da ampla gama de fatores que configuram o quadro epidemiológico atual das DCV.

Os fatores de risco comportamentais, ou condutas de risco, constituem metas primordiais da prevenção de enfermidades e a educação em saúde tem sido utilizada tradicionalmente para atingir essa meta. No entanto, dentro do marco mais amplo da promoção da saúde, as condutas de risco podem ser consideradas como respostas às condições de vida adversas e as ações devem incluir a criação de ambientes favoráveis à saúde ${ }^{11}$.

A Carta de Ottawa, fruto da I Conferência Internacional de Promoção da Saúde, define promoção da saúde como "o processo de capacitação da comunidade para atuar na melhoria da sua qualidade de vida e saúde, incluindo uma maior participação no controle deste processo" ${ }^{\prime 2}$.

A promoção da saúde visa assegurar a igualdade de oportunidades e proporcionar os meios para que indivíduos e comunidades tenham oportunidade de conhecer e controlar os fatores de- 
terminantes da sua saúde. Entre seus principais campos estão ambientes favoráveis a escolhas mais saudáveis, acesso à informação e educação em saúde, desenvolvimento de habilidades para uma vida saudável, bem como a reorganização dos serviços de saúde ${ }^{12}$.

Dentro desse contexto, programas de intervenção de base comunitária têm sido introduzidos em diferentes países desde o início da década de 70. O principal objetivo desses programas é diminuir a morbidade e a mortalidade por DCV através da redução dos fatores de risco cardiovascular nas comunidades, a partir da educação em saúde e das estruturas existentes na comunidade.

Diante disso, o objetivo do presente trabalho foi descrever experiências internacionais no combate às doenças cardiovasculares através de ações de promoção da saúde e prevenção integrada dos principais fatores de risco. Além disso, identificar as ações de vigilância sobre os fatores de risco para DCNT no Brasil e fazer uma breve reflexão sobre os desafios do Programa de Saúde da Família para o enfrentamento das DCV, uma vez que o objetivo dessa estratégia é a mudança do eixo da atenção para a promoção da saúde e a vigilância desta voltada às comunidades.

\section{Metodologia}

Trata-se de uma revisão bibliográfica de caráter descritivo-discursivo. Os dados foram obtidos através de busca nas principais bases de dados em saúde: Biblioteca Virtual e Editora do Ministério da Saúde; nas fontes de informação Ciências da Saúde em Geral - Lilacs, Medline, Scielo, Science Direct. Foram consultados ainda sites de órgãos oficiais internacionais: Organização Pan Americana de Saúde (OPAS) e Organização Mundial de Saúde (OMS).

Para a revisão, utilizaram-se os seguintes descritores: "heart health", "risk factors", "community-based intervention", "cardiovascular disease", "hypertension", "primary care", "health promotion" e seus correspondentes em português; de forma que a análise concentrou-se essencialmente em artigos de periódicos científicos da área de saúde, principalmente nas subáreas de medicina preventiva, saúde pública e epidemiologia.

A pesquisa ocorreu nos meses de junho a dezembro de 2008 e foram incluídos trabalhos publicados desde a década de 70, quando foram divulgadas as primeiras experiências de intervenção comunitária contra as DCV que inspiraram a formulação, pela Organização Mundial da Saú- de, de programas internacionais de combate às DCV baseados na ação comunitária.

$\mathrm{Na}$ busca bibliográfica foram obtidos 52 resultados, e após a leitura destes, selecionou-se para leitura mais detalhada e utilizou-se para a redação desta revisão bibliográfica 16 artigos científicos originais e três revisões narrativas, devido à sua maior relevância com o tema abordado. Os estudos foram selecionados de forma a construir uma perspectiva histórica dessas intervenções, destacando-se as experiências mais importantes relatadas na literatura. Os artigos foram incluídos após análise e classificação de acordo com categorias principais que enfocavam:

1. Divulgação de métodos e formulação de programas de intervenção que envolviam a mobilização de instituições comunitárias para a promoção de estilos de vida saudáveis em nível regional e nacional na prevenção das DCV;

2. Avaliação de resultados dos programas de intervenção comunitária para prevenção e tratamento das DCV a médio e longo prazo;

3. Aspectos teóricos e metodológicos dos programas de intervenção comunitária para a prevenção de DCV.

De forma complementar, utilizou-se ainda, artigos, documentos oficiais e teses relacionadas a temas atuais de políticas públicas relativas aos sistemas de vigilância dos fatores de risco para DCNT, assim como projetos demonstrativos de intervenção comunitária implantados no Brasil, de forma a construir um panorama geral das ações realizadas no sentido da prevenção primária dos fatores de risco para DCV no país nas últimas décadas. Considerando que no Brasil as políticas de saúde voltadas para a comunidade atualmente tem como eixo norteador a Estratégia de Saúde da Família (ESF), optou-se por selecionar também, trabalhos originais sobre a ação da ESF sobre as DCV e os desafios dessa estratégia no país.

\section{As intervenções comunitárias sobre as doenças cardiovasculares: uma perspectiva histórica}

As estratégias comunitárias partem da premissa de que ações de saúde pública têm um impacto potencial maior do que propostas em nível individual ${ }^{13}$. As estratégias visam a mudança de comportamentos de risco e sua sustentabilidade é dada por meio do envolvimento de organizações comunitárias. São baseadas na prevenção primária dos fatores de risco e na promoção da 
saúde através da mobilização comunitária, estratégias de comunicação de massa e atividades interativas, screenings populacionais e intervenções que visam mudanças ambientais ${ }^{14-27}$.

A primeira experiência comunitária descrita na literatura foi o programa Community Syndrome of Hypertension, Atherosclerosis and Diabetes (CHAD) desenvolvido como um projeto demonstrativo de cuidado primário em saúde orientado para a comunidade em Israel, iniciado em 1971. Tinha como principais objetivos: modificar as frequências de hipertensão arterial, de hipercolesterolemia e de sobrepeso na comunidade; identificar e tratar os indivíduos hipertensos, diabéticos, hipercolestrolêmicos e obesos; e modificar comportamentos comunitários relacionados à dieta, ao exercício físico e ao uso de tabaco. Os programas de educação em saúde foram baseados principalmente em aconselhamento direto com os profissionais. O CHAD produziu reduções significativas na prevalência de hipertensão $(20 \%)$, taxas de tabagismo (11\% nos homens) e sobrepeso (13\%). No entanto não foram encontradas diferenças nas taxas de mortalidade nessa população ${ }^{14}$.

O projeto Karélia do Norte (PKN) foi iniciado na província finlandesa da Karélia do Norte em 1972, em parceria com a OMS. Desde a década de 60 , a província tinha as maiores taxas de mortalidade por doenças isquêmicas do coração no mundo e uma população predominantemente rural e de baixo status socioeconômico. O objetivo principal do projeto foi testar a viabilidade e os efeitos de um programa comunitário para a prevenção de DCV por meio de mudanças no estilo de vida e nos fatores de risco, além de reduzir a alta mortalidade por DCV na área. O projeto incluiu programas de comunicação de massa, com ênfase nos fatores de risco e promoção da integração das intervenções com a atenção primária à saúde. Depois do êxito inicial e de importantes reduções, tanto dos fatores de risco como da mortalidade por DCV, iniciou-se um amplo e intenso trabalho nacional que incluiu em suas metas a diminuição da mortalidade causada pelas principais DCNT. Após os primeiros 25 anos da implantação do projeto, a mortalidade por DCV na Karélia do Norte decresceu significativamente: $68 \%$ por DCV em geral e $73 \%$ por cardiopatia coronariana. As mudanças ocorridas em toda a Finlândia foram igualmente importantes, havendo uma redução na mortalidade por DCV de 65\%. Análises têm demonstrado que boa parte da diminuição da mortalidade por DCV foi atribuída às mudanças da população em relação aos principais fatores de risco, ha- vendo evidências de que as modificações dietéticas foram o fator mais importante $\mathrm{e}^{15,16}$.

O projeto Stanford Three Community também iniciado em 1972, nos Estados Unidos, teve como objetivo a redução dos níveis de colesterol sérico, hipertensão arterial e tabagismo por meio de diferentes atividades de comunicação social (programas de televisão, rádio, jornais, cartas, panfletos) concentradas em ambientes como o lar, o trabalho e a comunidade e atividades educativas individuais e de grupo para indivíduos de alto risco. Os resultados foram favoráveis para tabagismo e colesterol sérico, sendo que o risco cardiovascular diminuiu em $17 \%$ nas comunidades de intervenção ${ }^{17}$.

O programa Franklin Cardiovascular Health, iniciado em 1974 em Franklin, Maine (EUA), teve a participação de 23 comunidades. O objetivo do programa foi reduzir o risco cardiovascular por meio da manutenção de valores adequados de $\mathrm{PA}$, colesterol e diminuição do tabagismo. O programa integrou a saúde pública e os serviços de saúde e as estratégias incluíram "screenings" populacionais, aconselhamento e participação comunitária. Pesquisas afirmam que a taxa de mortalidade em Franklin foi significativamente menor que o restante de Maine - 10\% para mortalidade total e $17 \%$ para mortalidade por DCV ${ }^{18}$.

Em 1978, no norte da Califórnia, o projeto Stanford Five City (SCP) testou um abrangente programa de organização comunitária e de educação em saúde nas mudanças favoráveis do risco cardiovascular - tabagismo, dieta, hipertensão arterial, sedentarismo e obesidade. O programa usou múltiplos métodos de educação para mudança dos fatores de risco comportamentais: mídia de massa (TV e rádio), mídia impressa através de correspondência, nos locais de trabalho e por meio dos serviços de saúde. Os resultados foram mudanças favoráveis no colesterol sérico $(2 \%)$, PA $(4 \%)$ e tabagismo $(13 \%)^{19}$.

O programa Minnesota Heart Health $(\mathrm{MHH})$ iniciado em 1981 também focou os fatores de risco para DCV como tabagismo, sedentarismo, sobrepeso e ingestão de álcool. As ações incluíram campanhas de comunicação de massa, educação e "screenings" sistemáticos dos fatores de risco, mudanças ambientais e educação dirigida aos jovens, aos adultos e aos profissionais de saúde. Não foram encontradas evidências de efeito significativo das intervenções sobre as $\mathrm{DCV}^{20}$.

O programa Pawtucket Heart Health (PHH) também iniciado em 1981 teve como foco as ações em organizações e campanhas comunitárias. $\mathrm{O}$ programa foi desenhado para, além de diminuir 
os fatores de risco comportamentais através da educação em saúde e do desenvolvimento de redes de apoio social, criar mecanismos de suporte para manutenção de hábitos saudáveis, por meio do fortalecimento das organizações comunitárias e de ações intersetoriais. As ações incluíram informações sobre alimentos pobres em gordura em mercados e restaurantes e programas nutricionais junto a livrarias públicas. Não houve diferença significante para pressão arterial, colesterol sérico e prevalência de tabagismo após a intervenção, apesar do risco de DCV ter diminuído cerca de $8 \%$ durante o programa de educação ${ }^{21}$.

Na Europa, o German Cardiovascular Prevention Study (GCP) foi um programa multicêntrico conduzido entre 1984 e 1991 em seis regiões da Alemanha Ocidental. O objetivo do programa era a redução, através da prevenção primária, de quatro principais fatores de risco cardiovascular - hipertensão, hipercolesterolemia, tabagismo e obesidade. Foi implementado um programa de prevenção multifacetado baseado em alimentação saudável, aumento da atividade física e realização de "screenings" para a detecção e o tratamento de indivíduos com HA e hipercolesterolemia. Contava com a parceria de instituições públicas e privadas e da comunidade médica; e as estratégias incluíram campanhas para promoção da alimentação saudável junto a festivais comunitários e a eventos públicos em escolas, mercados, e restaurantes. Foram oferecidos cursos e seminários para a redução de peso, e eventos esportivos recreativos para estimular a atividade física. Além disso, também foram implementados programas para a cessação do fumo. Foram encontrados modestos resultados na redução da pressão arterial (21\%), colesterol sérico (11\%) e taxas de tabagismo em homens $(9,7 \%)^{22}$.

No norte da Suécia foi conduzido na região de Norsjo, caracterizada pela maior incidência e mortalidade por DCV do país, um programa de prevenção através da mudança de fatores de risco (lipídeos plasmáticos, pressão arterial e tabagismo) iniciado em 1985. Foram conduzidos inquéritos anuais sobre os fatores de risco para DCV durante dez anos. Os indivíduos receberam aconselhamento sobre mudanças de estilo de vida e aqueles com alto risco tiveram tratamento médico. Houve diferenças significativas na pressão arterial sistólica e no colesterol total. Além disso, a mortalidade foi reduzida em 36\% na região de intervenção ${ }^{23}$.

No Canadá, a partir de 1986, foi lançado a Canadian Heart Health Initiative (CHHI), uma iniciativa federal com o objetivo de implementar programas de prevenção de DCV com foco na comunidade ${ }^{24}$. O programa Coeur en Santé Saint Henri, desenvolvido pelo departamento de saúde pública de Montreal, entre 1992 e 1995, envolveu uma comunidade de baixa renda, em Saint Henri, caracterizada por altas prevalências de tabagismo, sobrepeso e inatividade física. O principal objetivo do programa foi promover comportamentos saudáveis (controle da pressão arterial e colesterol total, aumento da atividade física, consumo de dietas com baixo teor de gordura e cessação do tabagismo) em indivíduos de 18 a 65 anos. As estratégias se concentraram em ações de educação nutricional e no combate ao tabagismo. No entanto, não foram encontrados resultados expressivos ${ }^{25}$.

O Quebec Heart Health Demonstrative Project foi um programa de prevenção de DCV baseada em ações comunitárias, conduzido entre os anos de 1992 e 1997, em diferentes províncias de Quebec, Canadá. O departamento de Saúde Pública de cada região foi responsável por definir os objetivos, a população alvo, as estratégias de intervenção e os métodos de avaliação. Os programas locais aderiam aos princípios básicos que dizem respeito à prevenção dos fatores de risco (tabagismo, dieta e sedentarismo), através de programas multifatoriais na comunidade e envolvendo diferentes segmentos da população e organizações locais. O projeto não alcançou resultados significativos nas mudanças dos fatores de risco comportamentais ${ }^{26}$.

Na Holanda, foi iniciado em 1998, o projeto Hartslag Limburg na região de Maastricht, que teve como objetivo reduzir a prevalência de DCV, encorajando os habitantes a aumentarem a atividade física, a reduzirem a ingestão de gordura, e a cessarem o tabagismo. O projeto integrou duas estratégias: uma direcionada à população em geral e aos grupos de baixo status socioeconômico; e outra focada nos indivíduos diagnosticados com DCV ou múltiplos fatores de risco para DCV. Foi realizado um trabalho intersetorial entre os conselhos da cidade de Maastricht e de quatro municípios adjacentes, o Instituto Regional de Saúde Pública de Maastricht, duas organizações de trabalho social da comunidade e a organização de cuidado à saúde local. De 1999 a 2003 foram implantadas 590 intervenções principais, sendo que $50 \%$ delas eram direcionadas a áreas de baixa renda. Exemplos de intervenções foram educação nutricional através de meios digitais, além de em espaços como em supermercados, parcerias público-privadas com programas de televisão, rotulagem de alimentos, áreas livres de tabaco, criação de campanhas e grupos de caminhada e ci- 
clismo, campanhas antitabaco, em adição a campanhas na rede de rádio e televisão local, artigos em jornais e distribuição de panfletos. Após cinco anos de intervenção, foram encontradas diferenças significativas nos valores de circunferência de cintura, índice de massa corporal (IMV) e pressão arterial na região de intervenção em comparação a regiões de referência ${ }^{27}$.

Os resultados dos programas de intervenção comunitária tiveram resultados bastante distintos nos países desenvolvidos. Muitos deles produziram resultados modestos nas mudanças de comportamentos de risco, em nível populacional. Algumas razões têm sido propostas para explicar o pequeno impacto de muitos programas de intervenções comunitárias em DCV. Elas incluem: o alcance limitado de sessões de aconselhamento individual e de grupos pequenos, uma baixa penetração desses programas de educação em muitos segmentos da população, fortes tendências seculares de aumento dos fatores de risco e as condições estatísticas de análise dos resultados ${ }^{13}$.

Dos programas de intervenção comunitária em DCV encontrados, o PKN foi aquele que obteve maior sucesso na diminuição da mortalidade por DCV, produzindo mudanças substanciais nos fatores de risco das DCV. Um ponto forte desse projeto foi a participação ativa de vários setores da comunidade, incluindo diversas organizações não governamentais, serviços de saúde e escolas, além da cooperação internacional da Organização Mundial da Saúde ${ }^{15}$.

Vários estudos de intervenção comunitária também têm sido desenvolvidos em países em desenvolvimento, incluindo Maurícios e China.

Maurícios, uma ilha multiétnica no Oceano Índico, teve um rápido e intenso processo de industrialização nas últimas décadas e enfrentava altas e crescentes taxas de mortalidade por DCV. Em 1987, o governo da ilha lançou um programa de intervenção sobre DCNT para prevenir e controlar essas doenças através de mudanças de estilos de vida. O objetivo do programa foi modificar os níveis de fatores de risco relacionados ao estilo de vida incluindo intolerância à glicose, HA, hiperlipidemia, obesidade, tabagismo, consumo de álcool e sedentarismo. Os componentes de prevenção primária incluíram o extensivo uso de mídia de massa, políticas fiscais e legislativas e atividades de educação sanitária na comunidade em geral, nas escolas e nos locais de trabalho. Resultados mostraram tendências favoráveis nos níveis de fatores de risco, com diferenças significativas na redução da prevalência de tabagismo (11\%), aumento da prática de atividade física moderada $(5,2 \%)$, redução do consumo de álcool $(23,8 \%)$, e redução do colesterol sérico $(18,9 \%)$ e HA $(2,9 \%)^{28}$.

Em Tianjin na China, onde a HA configurase como maior problema de saúde pública e onde há um alto consumo de sal, foi lançado em 1989 um programa de intervenção comunitária focado em ações que visavam a redução do consumo de sal e o controle do peso corporal nos indivíduos da comunidade, além da terapia adequada com anti-hipertensivos em pacientes portadores de HA. Inquéritos periódicos sobre os níveis de fatores de risco para DCV têm sido realizados desde a implantação das intervenções. Sete anos após estas, foram observadas mudanças favoráveis nos valores de pressão arterial e IMC (índice de massa corpórea), sobretudo nos indivíduos entre 45 e 64 anos. No entanto, as mudanças na pressão arterial, no IMC e no consumo de sal foram pequenas na comunidade em geral $^{29}$.

Revisão realizada por Gazeano et al. ${ }^{30}$ sobre o custo-efetividade dos programas de intervenção comunitária sobre DCNT concluiu que, apesar das pequenas reduções nos fatores de risco, tanto em países desenvolvidos quanto em países em desenvolvimento, elas são significativas na diminuição do risco-cardiovascular e poderiam ser custo-efetivas em países de baixa e média renda.

Baseados em experiências comunitárias bem sucedidas, recomendações tem sido propostas para o desenvolvimento de futuros programas, destacando-se a participação ativa da comunidade, a compreensão de suas necessidades e prioridades, a colaboração dos indivíduos, principalmente dos líderes e organizações comunitárias, a importância do envolvimento dos serviços de saúde locais, e a implementação de políticas nacionais que auxiliem escolhas saudáveis ${ }^{31,32}$.

\section{As ações comunitárias da Organização Mundial de Saúde}

Seguindo o exemplo do PKN e outros programas de promoção da saúde e de prevenção de enfermidades na América do Norte e Europa, a Organização Mundial da Saúde (OMS) iniciou o Programa Nacional Integrado de Intervenção contra Enfermidades Não Transmissíveis (CINDI, do inglês Countrywide Integrated Noncommunicable Diases Intervention Programe) que começou com programas nacionais de demonstração de 1985 a 1992 no América do Norte e Europa. Até o ano de 2002 a iniciativa possuía 105 áreas de demonstração em 29 países ${ }^{33}$. 
O projeto CARMEN (Conjunto de Ações Integradas para Redução Multifatorial de Enfermidades Não Transmissíveis) é uma iniciativa da Organização Pan Americana da Saúde (OPAS), correspondente ao CINDI para a América Latina e Caribe. O controle dos principais fatores de risco de risco é proposto através da ação simultânea sobre pelo menos três elos (tabagismo, dieta inadequada e sedentarismo) em três níveis de ação: político, comunitário e de serviços de saúde; e a incorporação das políticas públicas sistematicamente como nível de intervenção ${ }^{34}$.

Alguns pontos são considerados fundamentais para a concretização dos objetivos da iniciativa CARMEN, como a realização de inquéritos populacionais para a identificação dos principais fatores de risco cardiovascular, a abordagem dos fatores de risco de forma integrada e intersetorial e a reorganização dos serviços locais de Atenção Básica ${ }^{34}$.

A vigilância dos fatores de risco para doenças cardiovasculares e os projetos demonstrativos no Brasil.

Desde 2002, a OMS está envolvida no esforço mundial de priorizar a vigilância das DCNT, com foco nos principais fatores de risco tais como o tabagismo, a hipertensão arterial, a alimentação inadequada, a obesidade, o sedentarismo, entre outros, diante das experiências exitosas em vários países na redução da morbimortalidade por DCV por meio da vigilância e das ações integradas sobre os fatores de risco ${ }^{35}$.

O Brasil, por meio de diferentes inquéritos de saúde, vem construindo bases de dados para o monitoramento contínuo dos fatores de risco e de proteção das DCNT $^{4}$.

Realizadas em 2001/2 as Campanhas de detecção de suspeitos de hipertensão e diabetes no Brasil, parte do Plano de Reorganização da Atenção à Hipertensão Arterial e ao Diabetes Mellitus do Ministério da Saúde, configuraram a primeira iniciativa mundial de realização de uma campanha populacional massiva para o rastreamento de DCNT ${ }^{36}$.

Em 2002-2003, o Ministério da Saúde realizou nas capitais de 16 unidades da federação, o Inquérito Domiciliar sobre Comportamentos de Risco e Morbidade Referida de Doenças e Agravos não Transmissíveis. Esse inquérito coletou, entre outros dados, informações sobre hábito de fumar, peso e estatura referidos, consumo alimentar, atividade física, consumo de bebidas alcoólicas e diagnóstico médico de hipertensão arterial e

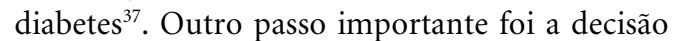
de incluir questões sobre hábito de fumar e ativi- dade física na Pesquisa Nacional por Amostragem de Domicílios do Instituto Brasileiro de Geografia e Estatística - IBGE, realizada em $2008^{38}$.

O Ministério da Saúde, por intermédio da Secretaria de Vigilância em Saúde e da Secretaria de Gestão Estratégica e Participativa e contando com o suporte técnico do Núcleo de Pesquisas Epidemiológicas em Nutrição e Saúde da Universidade de São Paulo - NUPENS/USP, ainda implantou, em 2006, o sistema VIGITEL - Vigilância de Fatores de Risco e Proteção para Doenças Crônicas por Inquérito Telefônico. Esse sistema permite o monitoramento contínuo e rápido dos fatores de risco para DCNT em todas as capitais brasileiras ${ }^{39}$.

Projetos demonstrativos de vigilância e condução de DCNT têm sido conduzidos no Brasil integrados à Estratégia de Saúde da Família. A iniciativa CARMEN é conduzida em municípios brasileiros desde 1998, com destaque para Goiânia - GO e Marília-SP ${ }^{4}$.

A implantação dessa iniciativa em Goiás fez surgir o Comitê Diretivo do CARMENT, com representantes de instituições parceiras como universidades, secretarias de saúde, educação e esporte e outras organizações de saúde. O comitê definiu dois critérios para adesão dos municípios: a decisão política do gestor local em adotar essa nova metodologia e a existência de Equipes de Saúde da Família (ESF), como forma de garantir a sustentabilidade da nova estratégia ${ }^{6}$.

Partindo dessa escolha, em 1999 foi realizado o diagnóstico sobre a prevalência de fatores de risco para doenças cardiovasculares, utilizando instrumentos recomendados em nível internacional pela Rede CARMEN das Américas ${ }^{40}$.

Os resultados evidenciaram alta prevalência de excesso de peso (44,2\%), hipertensão arterial $(32,9 \%)$ e tabagismo $(22,9 \%)$. A avaliação bioquímica evidenciou $8,1 \%$ de descontrole glicêmico, $45,5 \%$ de hipercolesterolemia e $17,7 \%$ de hipertrigliceridemia ${ }^{40}$.

Com base nesses dados, o Comitê CARMENT propôs para o Distrito Sanitário Leste as ações de intervenção, com a participação da comunidade. Integram essas ações a capacitação dos profissionais de saúde para diagnóstico precoce de fatores de risco para as DCNT, a abertura de espaços de lazer para o estímulo de exercícios físicos regulares, a proposição de uma investigação, junto a crianças e adolescentes da rede de ensino, buscando a detecção e a intervenção precoce sobre os fatores de risco presentes. Houve a interiorização da proposta com a implantação, em Firminópolis e no município de Quirinópolis, da iniciativa CARMENT ${ }^{4}$. 
A experiência acumulada e a interiorização da iniciativa para outros municípios permitiu com que o Estado fosse contemplado, em 2002, pelo Ministério da Saúde em conjunto com a OPAS/OMS, com o projeto de vigilância epidemiológica "Mega country", que tem como base metodológica a implantação de um sistema de vigilância e monitoramento de fatores de risco para DCNT - "STEPwise approach", da OMS, destinado a países com mais de 100 milhões de habitantes ${ }^{4,40}$

A experiência da implantação da Iniciativa CARMENT em Goiás resultou em iniciativas intersetoriais em parceria com a Faculdade de $\mathrm{Nu}$ trição, da Universidade Federal de Goiás, para a realização do primeiro diagnóstico sobre fatores de risco em escolares de 7 a 14 anos, por meio do Projeto Carminho ${ }^{41}$.

Através de inquérito realizado em 2004, para a avaliação dos resultados da estratégia em Goiânia sobre os fatores de risco cardiovascular, foram encontradas diminuições relativas significantes em ambos os sexos, nas prevalências de tabagismo $(4,8 \%)$, hiperglicemia $(3,7 \%)$ e trigliceridemia $(4,4 \%)$. No entanto, não foram encontradas diferenças significativas nos níveis de pressão arterial e sobrepeso/obesidade e houve uma tendência de aumento do sedentarismo ${ }^{42}$.

\section{A Estratégia de Saúde da Família: uma breve reflexão sobre os desafios no en- frentamento das DCV}

Analisando tanto as experiências internacionais quanto as políticas de promoção da saúde e prevenção de DCNT no Brasil, observa-se uma grande ênfase na intervenção de base comunitária e o papel protagonista dos serviços de Atenção Básica nesse processo.

Isso provavelmente se deve ao fato de que a Atenção Básica "caracterizada por um conjunto de ações de saúde, no âmbito individual e coletivo, que abrangem a promoção e a proteção da saúde, a prevenção de agravos, o diagnóstico, o tratamento, a reabilitação e a manutenção da saúde, é desenvolvida por meio do exercício de práticas dirigidas a populações de territórios bem delimitados" ${ }^{43}$. O território compreende o espaço onde vivem os grupos e que abriga suas relações e condições relacionadas à subsistência: trabalho, renda, habitação e acesso à educação. Além disso, nesse espaço consideram-se o saber preexistente e a cultura dos indivíduos, suas concepções sobre saúde e doença, família e sociedade, sendo local privilegiado para a ação em saúde porque nele consegue-se definir um conjunto de problemas e prioridades, e os recursos disponíveis para atender às necessidades de saúde de cada comunidade $^{44}$.

A Saúde da Família, estratégia priorizada pelo Ministério da Saúde para organizar a Atenção Básica e reestruturar o Sistema Único de Saúde (SUS), mostra-se como o modelo mais provável para alterar a realidade das DCV. Isso porque permite a aproximação dos indivíduos, do seu coletivo e de suas interações sociais, colaborando para a mudança de seus comportamentos e hábitos de vida ${ }^{6}$.

No Brasil, a iniciativa CARMENT - GO, implantada nas regiões onde há equipes da Estratégia Saúde da Família, indica que o novo modelo facilita a atenção, o controle dos fatores de risco e as ações de promoção da saúde ${ }^{6}$.

No entanto, estudos que a avaliaram as ações da ESF sobre a Hipertensão Arterial encontraram que apesar da estratégia ter aumentado o acesso dos portadores aos serviços de saúde, há uma baixa adesão ao tratamento e a permanência de uma alta prevalência de fatores de risco na comunidade. Esses resultados foram atribuídos principalmente a uma educação em saúde secundária e superficial, sem apreensão das dimensões psicossociais e culturais do processo saúde-doença-cuidado; a falta de alimentação do banco de dados HiperDia, a ausência de uma coordenação geral do programa nos municípios e a insipiência de ações baseadas na análise da situação de saúde dos hipertensos ou a programação de ações voltadas para os portadores desta enfermidade ${ }^{45-49}$.

As ações de base comunitária têm como elemento importante assegurar-se de que as organizações e os profissionais envolvidos sejam capacitados e recebam assistência técnica para a avaliação das necessidades das comunidades e para a implementação de estratégias integrais para intervenção sobre os fatores de risco comunitários, considerando as condições sociais e econômicas, tais como: a pobreza, o emprego, a composição familiar, o ambiente físico, as práticas, as normas e os valores, a urbanização e o acesso a bens e serviços ${ }^{34}$.

No entanto, o que se constata atualmente é que os profissionais de saúde que trabalham na Atenção Básica têm uma formação e um perfil inadequados para uma atuação na perspectiva da atenção integral à saúde e da implementação de práticas que contemplem ações de promoção, proteção, prevenção e atenção precoce ${ }^{50}$. Trabalhos que avaliaram o perfil e o conhecimento dos 
profissionais da Atenção Básica e Saúde da Família e diferentes atores envolvidos na construção da política de saúde local mostraram uma inadequação do conhecimento sobre os princípios e os objetivos do SUS e da ESF ${ }^{51,52}$.

A integralidade, um dos princípios doutrinários do SUS, têm sido um dos maiores desafios da saúde no Brasil, e a opção de ações baseadas em especialidades é responsável pela fragmentação dos trabalhos desenvolvidos na Atenção Básica. Além disso, constituem-se entraves às práticas integrais de saúde, o distanciamento entre o profissional e o usuário e as práticas de saúde orientadas por tecnologias não flexíveis e desvinculadas da realidade dos indivíduos ${ }^{44,53}$.

Nesse contexto, é relevante repensar a formação dos profissionais de saúde, que deve ser concebida de acordo com o Sistema Único de Saúde, baseada na gestão colegiada, na multiprofissionalidade e na alteridade, e realizada através de uma educação crítico-reflexiva, problematizadora, onde o ensino esteja em consonância com o serviço ${ }^{53,54}$.

Além da formação dos profissionais de saúde, outros elementos podem ser colocados como fundamentais para o sucesso da ESF, como a estrutura promotora de saúde. Estudo realizado por Campos ${ }^{55}$, em parceria com o Ministério da Saúde e a OPAS, que analisou várias experiências de promoção da saúde no âmbito da ESF, mostrou que fatores externos à ESF apresentaram-se como marcadamente necessários para que as iniciativas de promoção se desenvolvessem. Um ambiente político favorável, o empenho dos gestores locais, a presença de redes sociais atuantes no território e as ações intersetoriais mostraramse peças-chave nesse processo.

A intersetorialidade é defendida por outros autores como elo fundamental entre a promoção da saúde e a ESF, sendo que a última se constituiria de recurso para os profissionais lidarem com demandas intersetoriais por se configurar o ponto de articulação para o trabalho intersetorial ${ }^{56}$.

Talvez o maior desafio da ESF se deva ao seu papel de mudança do modelo de assistência tradicional, médico-medicamentoso e hospitalocêntrico. A "Saúde da Família é compreendida como estratégia principal para a mudança desse modelo (tradicional), que deverá se integrar a todo contexto de reorganização do sistema de saúde" 43 sendo o elo integrador e focalizador das políticas públicas nacionais e a comunidade.

\section{Considerações finais}

As DCV estão entre as principais causas de morte no Brasil e no mundo, sendo responsáveis por altos custos diretos e indiretos. A alta prevalência das DCNT, em especial as DCV, tem imposto desafios para o setor saúde e para as políticas públicas envolvidas no combate aos seus fatores de risco.

Estratégias de saúde pública, como as intervenções comunitárias, têm sido implantadas em vários países desenvolvidos e em desenvolvimento, como forma de enfrentamento das DCV a partir do enfoque de risco, traduzindo a tentativa de mudança do modelo de assistência prestada à população, fortalecendo os princípios da prevenção e da promoção, através de ações integradas e intersetoriais, com a participação efetiva da comunidade.

No Brasil, a implantação de sistemas de vigilância desses fatores está em consonância com esse novo enfoque. No entanto, muitos desafios permanecem no enfrentamento das DCV, principalmente no que diz respeito à integração das políticas públicas aos serviços de saúde. Nesse sentido, a atenção básica mostra-se como peça fundamental de elo entre políticas de saúde e a comunidade, e a ESF como estrutura potencial na consolidação da promoção da saúde.

\section{Colaboradores}

AG Ribeiro idealizou o trabalho, participou da pesquisa bibliográfica, metodologia, analise e discussão dos dados e redação do artigo; RMM Cotta idealizou, orientou e coordenou o trabalho, da pesquisa bibliográfica, metodologia; e, SM Ribeiro participou da analise e discussão dos dados, redação e correção do artigo.

\section{Agradecimentos}

Os estudos realizados foram financiados pela Fundação de Amparo à Pesquisa de Minas Gerais (Fapemig). 


\section{Referências}

1. Organização Pan-Americana da Saúde (OPAS). Doenças crônico-degenerativas e obesidade: estratégia mundial sobre alimentação saudável, atividade física e saúde. Brasília: Organização Pan-Americana da Saúde (OPAS); 2003

2. Brasil. Ministério da Saúde (MS). Hipertensão Arterial Sistêmica. Brasília: Ministério da Saúde (MS); 2006

3. DATASUS [homepage na internet]. Indicadores dados básicos - Brasil - 2007 [acesso em 30 abr 2008]. Disponível em: http://tabnet.datasus.gov.br/ cgi/idb2007/matriz.htm\#mort.

4. Malta DC, Cezario AC, Moura L. A construção da vigilância e prevenção das doenças crônicas não transmissíveis no contexto do Sistema Único de Saúde. Epidemiol Serv Saúde 2006; 15(3):47-65.

5. Brasil. Ministério da Saúde (MS); Organização PanAmericana da Saúde (OPAS). Avaliação do Plano de Reorganização da Atenção à Hipertensão Arterial e ao Diabetes Mellitus no Brasil. Brasília: Ministério da Saúde (MS); 2004.

6. Organização Pan-Americana da Saúde (OPAS). Ex periências e Desafios da Atenção Básica e Saúde Fami liar: caso Brasil. Brasília: Organização Pan-Americana da Saúde (OPAS); 2004.

7. McQueen DV. Continuing Efforts in Global Chronic Disease Prevention. Prev Chronic Dis [serial on the Internet] 2007 April [cited 2008 Mar 03]; 4(2): [about 21 p.]. Available from: http://www.pubmedcentral nih.gov/articlerender.fcgi?artid=1893120.

8. Brasil. Ministério da Saúde (MS). Prevenção Clínica de Doenças Cardiovascular, Cerobrovascular e Renal Crônica. Brasília: Ministério da Saúde (MS); 2006.

9. V Diretrizes Brasileiras de Hipertensão Arterial. Arq Bras Cardiol [periódico na Internet] 2007 Set [acessado 2009 ago 11]; 89(3): [cerca de 55 p.]. Disponível em: http://www. scielo.br/scielo.php?script=sci arttext\&pid=S0066-82X2007001500012\&lng=en. doi 10. 1590/S0066-782X2007001500012.

10. Buss PM. Promoção da Saúde da Família. Rev Bras Saúde Família 2002; 2(6):50-63.

11. Organização Mundial da Saúde (OMS). Glossário de Promoção da Saúde. Geneva: Organização Mundial da Saúde (OMS); 1988.

12. Organização Mundial da Saúde (OMS). Carta de Ottawa. In: Brasil. Ministério da Saúde, Fundação Oswaldo Cruz (Fiocruz). Promoção da Saúde: Carta de Ottawa, Adelaide, Sundsvall e Santa Fé de Bogotá. Brasília: Ministério da Saúde (MS)/IEC; 1986

13. Parker DR, Assaf AR. Community Interventions for Cardiovascular Disease. Prim Care Clin Office Pract 2005; 32(4): 865-881.

14. Abramson JH, Gofin J, Hopp C, Schein MH, Naveh P. The CHAD program for the control of cardiovascular risk factors in a Jerusalem community: a 24 year retrospect. Isr J Med Sci 1994; 30(1):108-119.

15. Puska P. Successful prevention on non-communicable diseases: 25 year experiences with North Karelia Project in Finland. Public Health Med 2002; 4(1):5-7.

16. Puska P, Salonen JT, Nissinen A, Tuomilehto J, Vartiainen E, Korhonen H, Tanskanen A, Rönnqvis P, Koskela K, Huttunen J. Change in risk factors for coronary disease during 10 years of a community intervention programme (North Karelia project). Br Med J 1983; 287(17):1840-1844.
17. Farquhar JW. The community-based model of lifestyle intervention trials. Am J Epidemiol 1978 ; 108(2):103-111.

18. Record NB, Harris DE, Record SS, Gilbert-Arcari J, DeSisto M, Bunnell S. Mortality impact of an integrated community cardiovascular health program. Am J Prev Med 2000; 19(1):30-38

19. Farquhar JW, Fortaman SP, Flora JA, Taylor CB, Haskell WL, Williams PT, Maccoby N, Wood PD. Effects of community-wide education on cardiovascular disease risk factors: The Stanford Five-CityProject. JAMA 1990; 264(3):359-365.

20. Luepker RV, Murray DM, Jacobs DR, Mittelmark MB, Bracht N, Carlaw R, Crow R, Elmer P, Finnegan J, Folsom AR. Community education for cardiovascular disease prevention: risk factor changes in the Minnesota Heart Health Program. Am Public Health 1994; 84(9):1383-1393.

21. Carleton RA, Lasater TM, Assaf AR, Feldman HA Mckinlay S, Pawtucket Heart Health Program Writing Group. The Pawtucket Heart Health Program: Community changes in cardiovascular risk factors and projected disease risk. Am J Public Health 1995; 85(6):777-785.

22. Hoffmeister H, Mensik GBM, Stolzenberg H, Hoeltz J, Kreuter H, Laaser U, Nussel E, Hullemann KD, Troschke JV. Reduction of Coranary Heart Disease Risk Factors in the German Cardiovascular Prevention (GCP) study. Prev Med 1996; 25(2):316-323.

23. Weinehall L, Hellsten G, Boman K. Prevention of cardiovascular disease in Sweden: the Norsjo community intervention programmed motives, methods and intervention components. Scand J Public Health 2001; 56(Supl):13-20.

24. Canadian Heart Health Initiative [homepage on Internet]. Process Evaluation of the Demonstration Phase - Conference of Principal Investigators of Heart Health 2001 [cited 2008 May 20]. Available from: http://www.med.ca/chhdbc/

25. O’Loughlin JL, Paradis G, Gray-Donald K, Renaud L. The impact of a community-based heart disease prevention program in low-income, inner-city neighborhood. Am J Public Health 1999; 89(12): 1819-1826.

26. Huot I, Paradis G, Ledoux M. Effects of the Quebec Heart Health Demonstration Project on adult dietary behaviours. Prev Med 2004; 38(2):137-148.

27. Schuit AJ, Wendel Vos GCW, Verschuren WMM, Ronckers ET, Van Ree J, Ruland EC. Effect of 5 Year Community Intervention Hartslag Limburg on Cardiovascular Risk Factors. Am J Prev Med 2006; 30(3):237-242.

28. Dowse GK, Gareeboo H, Alberti KGMM, Zimmet P, Tuomilehto J, Purran A, Fareed D, Chitson P, Collins VR. Changes in population cholesterol and other cardiovascular risk factor levels after five years of the non-comunicable disease intervention programme in Mauritius. British Med J 1995; 311(11):1255-1259.

29. Yu Z, Song G, Guo Z, Zheng G, Tian H, Vartiainen $\mathrm{H}$, Puska P, Nissinen A. Changes in blood pressure, body mass index, and salt consumption in a Chinese population. Prev Med 1999; 29(3):165-172.

30. Gazeano TA, Galea G, Reddy KS. Scaling up interventions for chronic disease prevention: the evidence. Lancet 2007; 370(9603):1939-1946. 
31. Pearson TA, Walls S, Lewis C. Dissecting the "black box" of community intervention: lessons from community-wide cardiovascular disease prevention programs in the US and Sweden. Scand J Public Health 2001; 29(Supl. 56):69-78.

32. Schooler C, Farquhar JW, Fortmann SP. Synthesis of ûndings and issues from community prevention trials. Ann Epidemiol 1997; (Supl. 7):S54-68.

33. Leparski E, Nussel E, editors. Countrywide Integrated Noncommunicable Diseases (CINDI) Intervention Programme - Protocol and guidelines for monitoring and evaluation procedures. Berlin: SpringerVerlag; 1987.

34. Brasil. Ministério da Saúde (MS). Secretaria de Políticas de Saúde. Departamento de Atenção Básica. Conjunto de ações para redução multifatorial das enfermidades não transmissíveis - Projeto Carmen Brasil - Protocolo Nacional. Brasília: Ministério da Saúde (MS); 2000.

35. Organização Mundial da Saúde (OMS) [homepage na Internet]. Conjunto de Acciones para Reducción Multifactorial de Enfermedades No Transmisibles (CARMEN) [acessado 2008 jul 1]. Disponível em: http://www.who.int/hpr/globalforum/regional. networks.shtml\#CARMEN.

36. Toscano CM. As campanhas nacionais para detecção das doenças crônicas não-transmissíveis e hipertensão arterial. Cien Saude Colet 2004; 9(4):885895.

37. Brasil. Ministério da Saúde (MS). Secretaria de Vigilância em Saúde. Instituto Nacional do Câncer. Inquérito domiciliar sobre comportamento de risco e morbidade referida de doenças e agravos não transmissíveis: Brasil, 15 capitais e Distrito Federal, 20022003. Rio de Janeiro: Ministério da Saúde (MS); 2004.

38. Malta DC. Agenda de prioridades de vigilância e prevenção das doenças crônicas não-transmissíveis e Seminário Nacional de Doenças e Agravos NãoTransmissíveis. In: Anais do Seminário Nacional de Vigilância em Doenças e Agravos Não-Transmissiveis e Promoção da Saúde; 2005 set 20-22; Brasília, Brasil. Brasília: Ministério da Saúde (MS); 2006. p. 16-19.

39. Brasil. Ministério da Saúde (MS). Secretaria de Vigilância em Saúde. Vigitel Brasil 2006: vigilância de fatores de risco e proteção para doenças crônicas por inquérito telefônico. Brasília: Ministério da Saúde (MS); 2007.

40. Carnelosso MLC, Barbosa MA, Porto CC, Silva SA, Carvalho MM, Oliveira ALI. Prevalência de Fatores de Risco para Doenças Cardiovasculares na região leste de Goiânia - Goiás. Cien Saude Colet [periódico na internet]. 2007 [acessado 2011 nov 8]; 15 (Supl. 1):[cerca de 8 p.]. Disponível em: http://www. abrasco.org.br/cienciaesaudecoletiva/artigos/ artigo_int.php?id_artigo $=1824$

41. Monego EM. Determinantes de Risco para doenças cardiovasculares em Escolares do município de Goiânia [tese]. Goiânia (GO): Programa Multiinstitucional de Pós-Graduação em Ciências da Saúde, Convênio Centro Oeste/Universidade Federal de Goiás (UFG), Universidade Federal de Mato Grosso do Sul (UFMS); 2005.
42. Carnelosso MLC. Iniciativa Carment: resultados e tendências das prevalências dos fatores de risco cardiovasculares [tese]. Goiânia (GO): Programa Multiinstitucional de Pós-Graduação em Ciências da Saúde, Convênio Centro Oeste/Universidade de Brasília (UNB), Universidade Federal de Goiás (UFG), Universidade Federal de Mato Grosso do Sul (UFMS); 2008.

43. Brasil. Ministério da Saúde (MS). Secretaria de Atenção à Saúde. Departamento de Atenção Básica. Política Nacional da Atenção Básica. Brasília: Ministério da Saúde (MS); 2006.

44. Campos CEA. O desafio da integralidade segundo as perspectivas da vigilância da saúde e da saúde da família. Cien Saude Colet 2003; 8(2):569-584.

45. Paiva DCP, Berbusa APS, Escuder MML. Avaliação da assistência ao paciente com diabetes e/ou hipertensão pelo Programa Saúde da Família no Município de Francisco Morato, São Paulo, Brasil. Cad Saude Publica 2006; 22(2):377-385.

46. Alves VS, Nunes MO. Educação em saúde na atenção médica ao paciente com hipertensão arterial no Programa Saúde da Família. Interface Comun Saúde Educ 2006; 10(19):131-147.

47. Araujo JC, Guimarães AC. Controle da Hipertensão Arterial em uma Unidade de Saúde da Família. Rev Saude Publica 2007; 41(3):368-374.

48. Toledo MM, Rodrigues SC, Chiesa AM. Educação em saúde no enfrentamento da hipertensão arterial: uma nova ótica para um velho problema. Texto Contexto - Enferm 2007; 16(2):233-238.

49. Costa JMBS, Silva MRF, Carvalho EF. Avaliação da Implantação da Atenção à Hipertensão Arterial pelas Equipes de Saúde da Família no Município de Recife - PE. Cien Saude Colet [periódico na internet]. 2011 [acessado 2011 nov 8];16(2): [cerca de 9 p.]. Disponível em: http://www.scielosp.org/pdf/ csc/v16n2/v16n2a26.pdf

50. Gil CRR. Formação de recursos humanos em saúde da família: paradoxos e perspectivas. Cad Saude Publica 2005; 21(2):490-498.

51. Cotta RMM, Mendes FF, Muniz JN. Descentralização das Políticas Públicas de Saúde - Do imaginário ao Real. Viçosa: Editora UFV; 1998.

52. Cotta RMM, Azeredo CM, Schott M, Martins PC, Franceschini SCC, Priori SE. Sobre o conhecimento e a consciência sanitária brasileira: o papel estratégico dos profissionais e usuários no sistema sanitário. Rev Med Minas Gerais 2006; 16(1):2-8.

53. Cotta RMM, Gomes AP, Maia TM, Magalhães KA, Marques ES, Siqueira-Batista R. Pobreza, injustiça, e desigualdade social: repensando a formação de profissionais de saúde. Rev Bras Educ Med 2007; 31(3):278-286

54. Ceccim RB, Feuerwerker LCM. Mudança na graduação das profissões de saúde sob o eixo da integralidade. Cad Saude Publica 2004; 20(5):1400-1410.

55. Campos FC. Promoção de Saúde na Atenção Básica no Brasil. Belo Horizonte: Núcleo de Estudos em Saúde Coletiva Faculdade de Medicin, Universidade Federal de Minas Gerais (UFMG), Fundação de Desenvolvimento da Pesquisa (FUNDEP); 2005. [Relatório OPAS: BR/CNT/0500054.001].

56. Cohen SC, Cynamon SE, Kligerman DC, Assumpção RF. Habitação saudável no Programa Saúde da Família (PSF): uma estratégia para as políticas públicas de saúde e ambiente. Cien Saude Colet 2004; 9(3):807-813. 